\title{
Loop gravity from a spinorial action
}

\section{Wolfgang Wieland*}

Institute for Gravitation and the Cosmos, Penn. State University

E-mail: wmwielandegmail.com

Spinors have a wide range of applications, from quantum mechanics to particle physics, quantum information and general relativity [1]. In this talk, I will argue that they are useful also for discretized gravity, and present a version of first-order Regge calculus with spinors as the fundamental configuration variables [2]. The underlying action describes a mechanical system with finitely many degrees of freedom, the system has a Hamiltonian, and local gauge symmetries. I will derive the resulting quantum theory, and explain the relation to loop quantum gravity [3].

References

[1] R. Penrose and W. Rindler, Spinors and Space-Time, Two-Spinor Calculus and Relativistic Fields, vol. 1. Cambridge University Press, Cambridge, 1984.

[2] W. M. Wieland, Hamiltonian spinfoam gravity, Class. Quantum Grav. 31 (2014) 025002, arXiv:1301.5859.

[3] C. Rovelli, Quantum Gravity, Cambridge University Press, Cambridge, November, 2008

Frontiers of Fundamental Physics 14 - FFP14,

15-18 July 2014

Aix Marseille University (AMU) Saint-Charles Campus, Marseille

${ }^{*}$ Speaker. 